03,16

\title{
Характеристика микродеформационных полей в кристаллах разбавленных магнитных полупроводников ZnSe:Co по данным нейтронной дифракции
}

\author{
(C) В.И. Максимов, Е.Н. Максимова, Т.П. Суркова, В.Д. Пархоменко
}

Институт физики металлов им. М.Н. Михеева УрО РАН,

Екатеринбург, Россия

E-mail: kokailo@rambler.ru

Поступила в Редакцию 19 апреля 2021 г.

В окончательной редакции 19 апреля 2021 г.

Принята к публикации 26 апреля 2021 г.

\begin{abstract}
Методом рассеяния тепловых нейтронов охарактеризована реальная структура объемных кубических кристаллов $\mathrm{Zn}_{1-x} \mathrm{Co}_{x} \mathrm{Se}(x=0.01 ; 0.15)$. Из анализа профилей структурных пиков, измеренных в тангенциальных и радиальных направлениях на исследованных кристаллах, получены сведения о результирующем поле микродеформаций, обусловленном атомными смещениями. Обнаруженные признаки дестабилизации исходной решетки обоих кристаллов показывают, что повышение содержания Со в селениде цинка до уровней, близких к естественному пределу растворимости в соединении, приводит к качественным изменениям в характере дефектности структуры. В частности, возрастающая вероятность появления субструктур микроблоков при высоких уровнях легирования сопровождается преобладанием микродеформаций растяжения по всему объему кристалла.
\end{abstract}

Ключевые слова: разбавленные магнитные полупроводники, монокристаллы, дифракция нейтронов, реальная структура кристаллов, микродеформации.

DOI: 10.21883/FTT.2021.08.51155.092

\section{1. Введение}

Интерес к исследованиям свойств кубических полупроводниковых соединений $\mathrm{Zn}_{1-x} M e_{x} \mathrm{Se} \quad(M e-$ $3 d$-элемент), относящихся к разбавленным магнитным полупроводникам (РМП) с матрицами $\mathrm{A}^{2} \mathrm{~B}^{6}$, не ослабевает на протяжении нескольких последних десятилетий. Указанные материалы, широко применяемые в электронно-оптических устройствах [1-3] и перспективные для использования в элементах спинтроники [3-4], с развитием нанотехнологий становятся сегодня весьма привлекательными для исследования их свойств на объектах наномасштаба [5-8].

Понижение размерности РМП с матрицами $\mathrm{A}^{2} \mathrm{~B}^{6}$ позволяет достичь более высокого уровня легирования $3 d$-ионами, т.е. повысить предел растворимости магнитоактивной примеси в полупроводнике по сравнению с естественным для объемного материала. При этом развитие полупроводниковой лазерной техники требует совершенствования методик синтеза „рабочих элементов“, особенно в плане управления диффузионными процессами [9] и получившей признание и распространение технологии „горячего изостатического прессования“ (hot isostatic pressing) [10-11].

Легирование матриц $\mathrm{A}^{2} \mathrm{~B}^{6}$ ионами кобальта представляет особый интерес для изучения физики РМП. Согласно энергетическим диаграммам расщепления конфигурации $d^{7}[12-14]$, широко используемым для анализа результатов измерений физических свойств [14-15], основное состояние иона $\mathrm{Co}^{2+}$ в тетраэдрическом кубическом поле ${ }^{4} A_{2}$ - орбитальный синглет - в общем, теоретически не предполагает эффекта Яна-Теллера и связанных с ним аномалий, обусловленных возмущениями электронной структуры. По данным [14], параметры $a$ кристаллической решетки кубических фаз $\mathrm{Zn}_{1-x} \mathrm{Co}_{x} \mathrm{~S}$ и $\mathrm{Zn}_{1-x} \mathrm{Co}_{x} \mathrm{Se}$ не зависят от концентрации Со до уровней легирования $x=0.05-0.10$, но рентгеновские эмиссионные спектры кристаллов указанного состава показывают признаки искажений в решетке [16]. В то время как в кристаллах $\mathrm{Zn}_{1-x} \mathrm{Co}_{x} \mathrm{~S}$ и $\mathrm{Zn}_{1-x} \mathrm{Co}_{x} \mathrm{Se}$ обнаружены признаки доминирующих антиферромагнитных взаимодействий [15], более сильных, чем в аналогичных РМП с марганцем [17], исследования халькогенидов цинка с кобальтом выявляют на нанообъектах значительное разнообразие магнетизма в зависимости от содержания магнитоактивной примеси и от наносостояния образцов (порошки, слои, проволоки и др.) [5,7,18-21]. Изучение эффектов совместного легирования кобальта с другими $3 d$-ионами (особенно с $\mathrm{Fe}$ ) в полупроводниковых матрицах $\mathrm{A}^{2} \mathrm{~B}^{6}$ оказывается востребованным для развития лазерной техники, работающей в диапазоне инфракрасного излучения. Так солегирование халькогенидов цинка железом и кобальтом представляется многообещающим для преодоления проблем накачки, обусловленных спектральным положением уровней поглощения $\mathrm{Fe}^{2+}-$ предполагая инверсионные процессы энергопереноса между двумя ионами разных $3 d$-переходных металлов в полупроводнике [2]. 
При этом, особенно для случая „сильно легированных“ РМП, в стороне не может оставаться проблема изодиморфических рядов $\mathrm{ZnX}-\mathrm{MeX}$ (Me $3 d$-элемент), когда в случае $X=\mathrm{S}$, Se для „крайних составов“" характерны разные структуры [22-25]. Названная проблема для кобальтосодержащих халькогенидов, помимо своего фундаментального характера в рамках изучения предпереходных состояний при концентрационных фазовых превращениях, сегодня тесно связана с физикой нанокомпозитов. Для используемых халькогенидов переходных металлов в современных компактных энергоустройствах (батареях, аккумуляторах, конденсаторах и др.), перспективы прецизионной настройки электронной структуры рабочего наноматериала и его электрохимической кинетики так же открываются со-допированием $3 d$-ионов [26].

В настоящей работе анализируется структурное состояние монокристаллов $\mathrm{ZnSe}: \mathrm{Co}$, при этом наибольшее внимание уделяется именно случаю „сильного легирования“. Анализ структурного состояния проводился в данной работе методом дифракции тепловых нейтронов. Ранее в наших работах [27-28] было показано, что механизм возмущения кристаллической решетки исходного полупроводникового материала ионами двухвалентного кобальта при невысоком содержании примеси может проявляться через признаки низкочастотных динамических деформаций.

\section{2. Образцы и методика эксперимента}

Для проведения экспериментов были выращены монокристаллы $\mathrm{Zn}_{1-x} \mathrm{Co}_{x}$ Se с содержанием примеси $x=0.01$ и $x=0.15$. Кристаллы были получены из газообразной фазы. Образцы имели неправильную форму, близкую к сферической, со средним диаметром $\sim 0.5 \mathrm{~cm}$ в трех взаимоперпендикулярных направлениях. В обоих случаях, большие плоские грани образца соответствовали плоскостям $\{100\}$ или $\{110\}$ кубической ячейки кристалла.

Эксперименты по упругому рассеянию тепловых нейтронов проводились на многоканальном дифрактометpe для исследований монокристаллов. Длина волны падающих на образец нейтронов $(\lambda)$, сформированная двойным кристалл-монохроматором из пиролитического графита и деформированного германия, составляла $1.57 \AA$. Эффективная монохроматизация первичного пучка и оптимальный выбор длины волны нейтронов существенно повышает чувствительность нашей нейтронографической методики. Измерения картин нейтронной дифракции проводились при температуре $300 \mathrm{~K}$.

\section{3. Результаты эксперимента и их обсуждение}

Нейтронографическая аттестация исследованных в работе кристаллов $\mathrm{Zn}_{1-x} \mathrm{Co}_{x} \mathrm{Se}(x=0.01$ и $x=0.15)$ показала соответствие их основного структурного мотива структуре сфалерита. Наряду с верификацией кубической структуры, кривые отражения интенсивных брэгговских рефлексов показали наличие объемного фрагмента „совершенного“ кристалла в исследованных образцах.

В качестве наглядного примера, на рис. 1 приведены картины рассеяния тепловых нейтронов в основании брэгговского рефлекса (004), полученные методом $\omega$-сканирования от кристаллов $\mathrm{Zn}_{1-x} \mathrm{Co}_{x} \mathrm{Se}$ с уровнями легирования $x=0.15(a)$ и $x=0.01(b)$. Обе картины на рис. 1, $a$ и $b$ нормированы к пиковой интенсивности рефлекса, принятой равной 100 arb.units.

Напомним, при анализе результатов дифракционного эксперимента основные векторы, определяющие его геометрию, связаны между собой хорошо известным соотношением

$$
\boldsymbol{\kappa}=\left(\mathbf{B}_{\mathbf{h k l}}+\mathbf{q}\right),
$$

где $\boldsymbol{\kappa}$ - вектор рассеяния, $\mathbf{B}_{\mathbf{h k l}}$ - вектор узла обратной решетки, q - волновой вектор [27,29-30].

При надежно обнаруживаемых признаках диффузного рассеяния нейтронов на картинах отражения исследуемого кристалла, подобных представленным на рис. 1, когда вектор q перпендикулярен вектору $\mathbf{B}_{\mathbf{h k l}}$, методически необходимо вводить вектор, характеризующий атомные смещения в кристалле, обычно обозначаемый и [29-30]. В таком случае, наблюдаемый в окрестности основного структурного рефлекса дополнительный диффузный вклад обусловлен сдвиговыми смещениями ионов, когда вектор и параллелен вектору $\mathbf{B}_{\mathbf{h k l}}$. Это означает, что наблюдающийся дифракционный эффект обусловлен поперечными относительно вектора рассеяния нейтронов смещениями ионов в плоскости рассеяния кристалла. Поперечные смещения ионов кристаллической решетки обычно принято характеризовать двумя векторами: $\Delta \mathbf{g}$ - вектором протяженности неоднородности в пространстве переданного импульса при наличии искажений кристаллической решетки в данном кристаллографическом направлении и и - вектором атомных смещений в кристалле. Используя данные нейтрон-дифракционного эксперимента, можно оценить модули указанных векторов или, по крайней мере, выявить тенденции их изменений от выбранных внешних или внутренних воздействий на исследуемую систему. Среднее значение модуля вектора $\Delta \mathbf{g}$ допускается принять равным истинной полуширине диффузного вклада в рассеяние, интенсивность которого пропорциональна квадрату модуля вектора и [30].

В описанном отношении, экспериментальная ситуация на рис. $1, b$ достаточно подробно была разобрана в работах [27-28]. В частности на поведении параметров моделирования рассматриваемого диффузного вклада с температурой мы показали, что ионы $\mathrm{Co}^{2+}$, как и практически все ионы $3 d$-ряда, несферичность $d-$ оболочки которых обусловлена незаполненными электронными уровнями, индуцируют наноразмерные неоднородности 

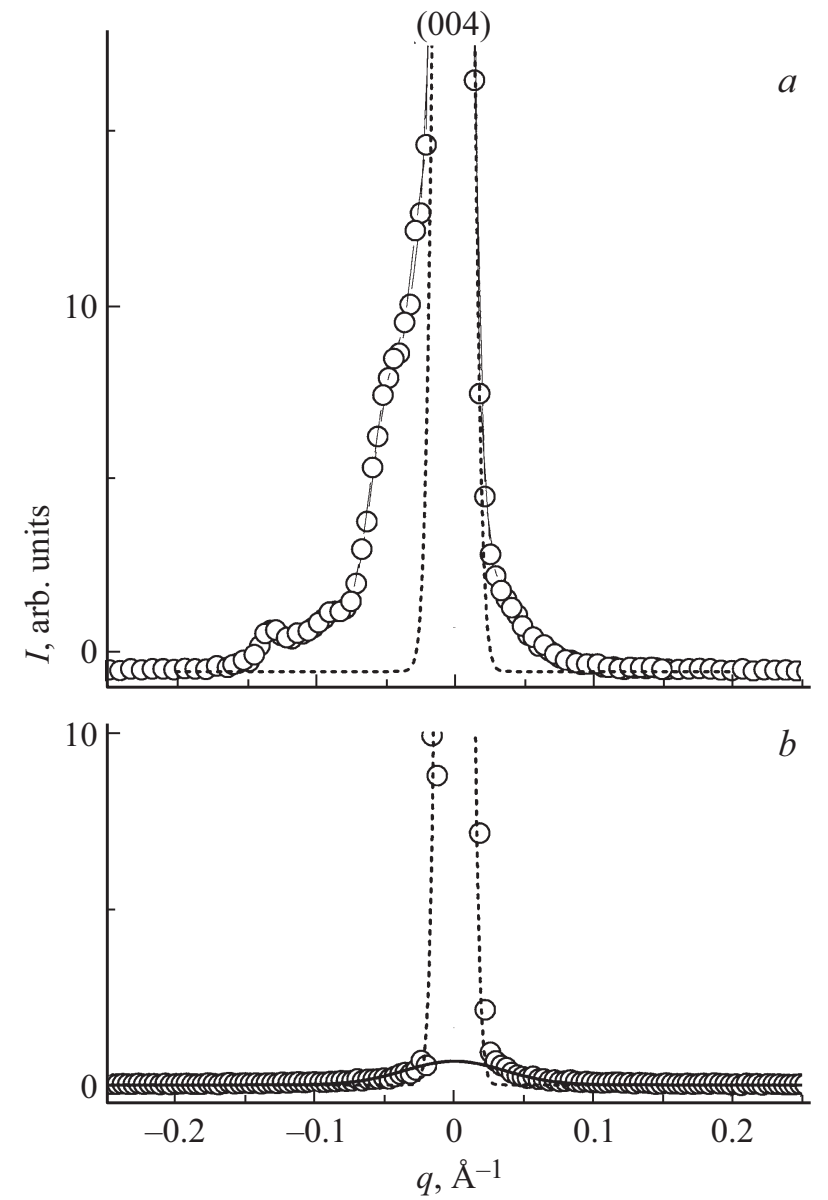

Рис. 1. Окрестность брэгговского рефлекса (400) вдоль тангенциального направления [010] в кристаллах $\mathrm{Zn}_{1-x} \mathrm{Co}_{x} \mathrm{Se}$ $x=0.15(a)$ и $x=0.01(b)$. Пунктир - профили рефлексов, сплошные линии - профили диффузного рассеяния. $T=300 \mathrm{~K}$

в структуре исходного полупроводникового кристалла. Но при этом, в отличие от случая легирования других элементов $3 d$-ряда $\mathrm{V}, \mathrm{Cr}, \mathrm{Fe}, \mathrm{Ni}[31]$, с охлаждением образца индуцированные кобальтом нанообласти повреждений структуры не изменяют своих размеров, но формирующие их амплитуды атомных смещений (так же как и, возможно, количество сдвиговых смещений в общей картине локальных возмущений) уменьшаются. Охарактеризованный таким образом на качественном уровне эффект указывает на динамическую природу проявляемой деформации.

Как наглядно показывают нормированные картины кривых отражения рис. 1, интенсивность эффектов дополнительного рассеяния (соответствующие профили на рисунке проведены непрерывной линией) в окрестности структурных рефлексов кристалла $\mathrm{Zn}_{1-x} \mathrm{Co}_{x} \mathrm{Se}$ с уровнем легирования $x=0.15$ на рис. $1, a$, в сравнении с данными рис. $1, b$, демонстрирующими похожий эффект рассеяния на неоднородностях в кристалле с содержанием кобальта $x=0.01$, возрастает почти на порядок.
Высота его максимума достигает порядка 10\% от максимума интенсивности структурного пика, а его форма, наблюдаемая в том же тангенциальном направлении, представляет ломаный, асимметричный профиль. Подобная форма кривой вполне отвечает рассеянию на микрокристаллических блоках, слабо разориентерованных относительно основного отражающего массива. Другими словами, данные рис. 1, $а$ указывают на сформированную субструктуру микрокристаллитов, появление которой, в сопоставлении с экспериментальными данными нейтронографии для случая более слабого легирования, вполне логично относить к усилению микроблочности.

Из самых общих соображений, увеличение в кубическом кристалле $\mathrm{ZnSe}$ содержания примеси Co, для селенида которого наиболее характерна принципиально отличающаяся от сфалерита гексагональная структура типа NiAs, неизбежно ведет к росту вероятности эффектов смещения и замещения ионов в процессе получения легированного кристалла. Поэтому обнаруженную нами в настоящей работе субструктуру сильно легированного кристалла $\mathrm{Zn}_{1-x} \mathrm{Co}_{x} \mathrm{Se}(x=0.15)$ следует считать возникающей благодаря формированию протяженных локальных скоплений статических дефектов.

На рис. 2-4 приведены картины нейтронного рассеяния кристалла $\mathrm{Zn}_{1-x} \mathrm{Co}_{x} \mathrm{Se}(x=0.15)$, сканированные в радиальных направлениях через узлы обратной решетки. Рефлексы (111), (200), (220), попадающие в нашем эксперименте в область наилучшего разрешения по отношению к нейтрон-оптическому диапазону поля зрения дифрактометра (рис. 2) неплохо описываются единственной функцией Гаусса, а наблюдающиеся при бо́льших углах скольжения - могут проявлять заметные и существенные отклонения от простого описания (рис. 3-4). Для моделирования аномальных форм профилей рефлексов сильно легированного кристалла $\mathrm{Zn}_{1-x} \mathrm{Co}_{x} \mathrm{Se}(x=0.15)$ в наибольшей степени подходит суммирование функций Гаусса (на рис. 3-4 результат фиттинга демонстрируют толстые непрерывные линии), а в отдельных случаях - введением асимметричных форм, составленных из полугауссианов (на рис. 3-4 моделирующие компоненты проведены пунктиром). Во всех случаях описания экспериментальных данных значения коэффициента детерминации $r^{2}$ были не хуже 0.97 .

Как было показано нами ранее в работах [32-34], обнаруживаемые аномальные формы профилей рефлексов сильно легированных $3 d$-ионами объемных кристаллов халькогенидов цинка свидетельствуют в пользу деформированного состояния, сформированного в поврежденной исходной структуре двойного полупроводника на базе корреляций между атомными смещениями. Профильный анализ рефлексов, сканированных вдоль радиальных направлений, характеризует неоднородное поле деформаций.

Как наглядно демонстрируют данные рис. 3-4, полуширины компонент соизмеримы друг с другом, что обуславливает их проявление только в „дальних углах“. Характер расщепления для всех измеренных рефлексов 


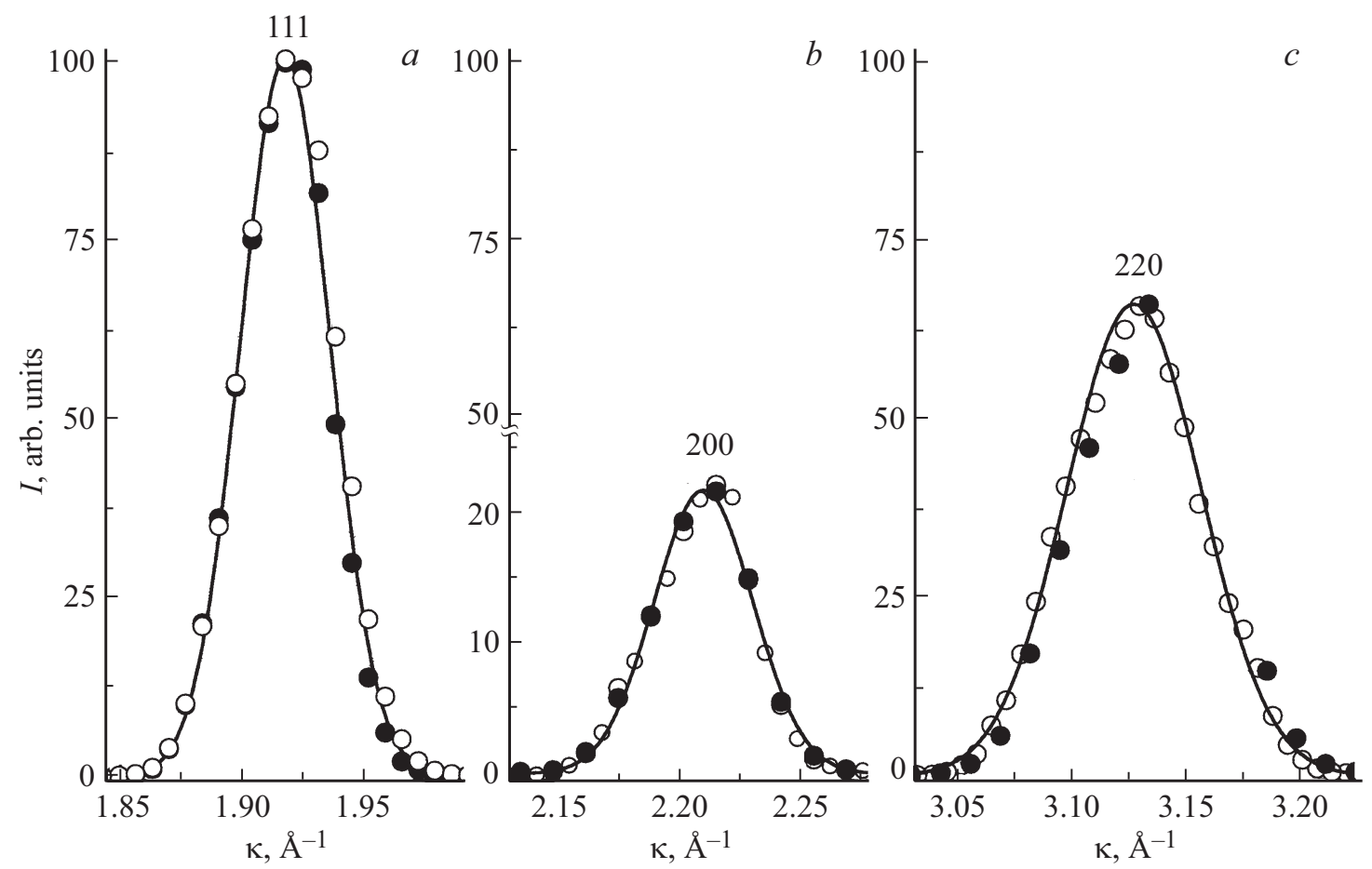

Рис. 2. Картины нейтронной дифракции монокристалла $\mathrm{Zn}_{1-x} \mathrm{Co}_{x} \mathrm{Se}(x=0.15)$, измеренные при $300 \mathrm{~K}$ относительно основных узлов обратной решетки в радиальных направлениях (соответственно, светлые и темные кружки): (111) и $(\overline{1} 11)(a),(200)$ и (020) (b), (022) и (220) (c).
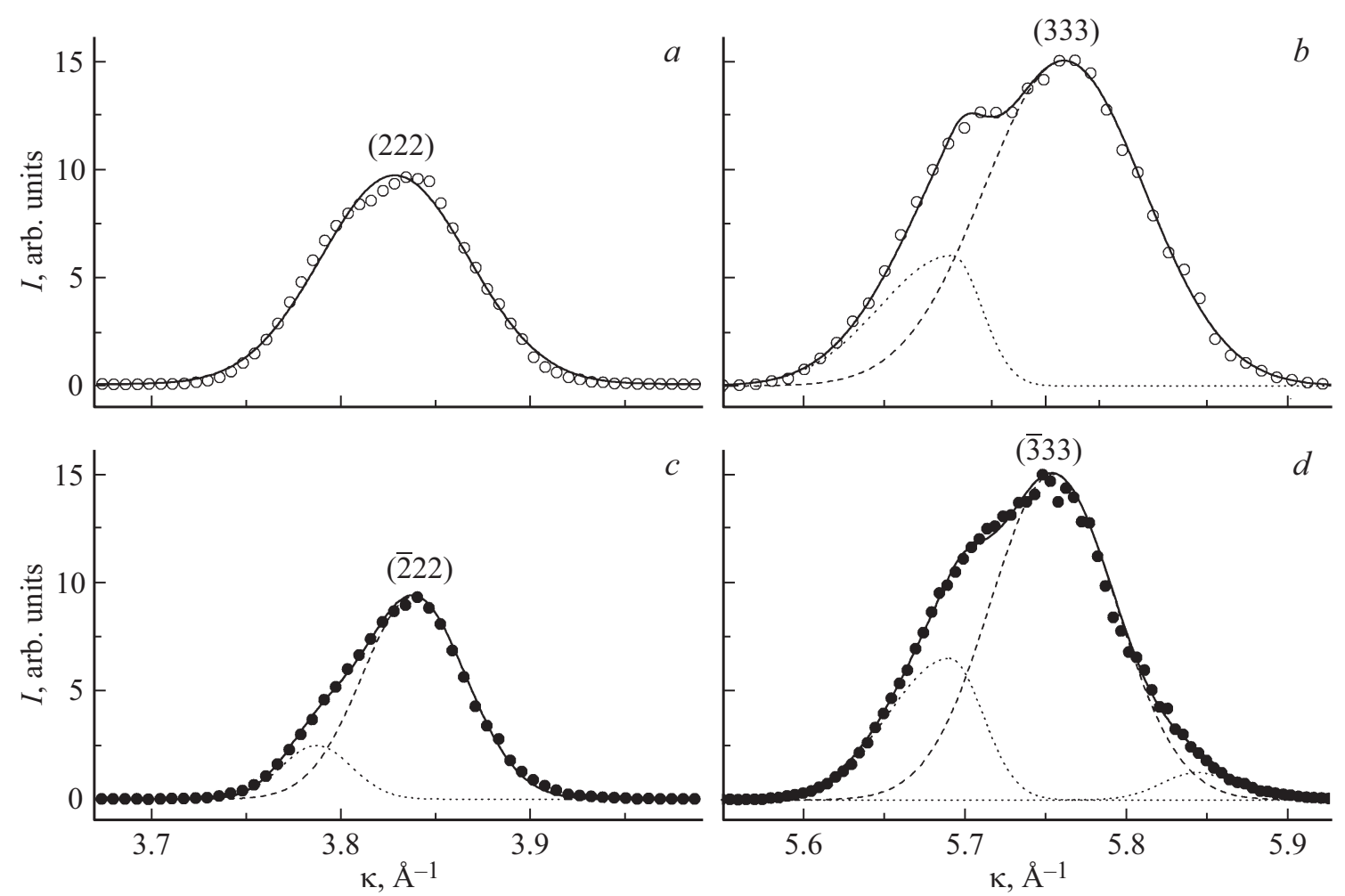

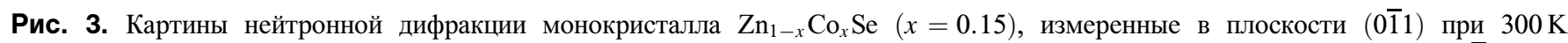
относительно основных узлов обратной решетки в радиальных направлениях: (222) (a) и (333) $(b)-$ светлые кружки, $(\overline{2} 22)(c)$ и $(\overline{3} 33)(d)$ - темные кружки. 

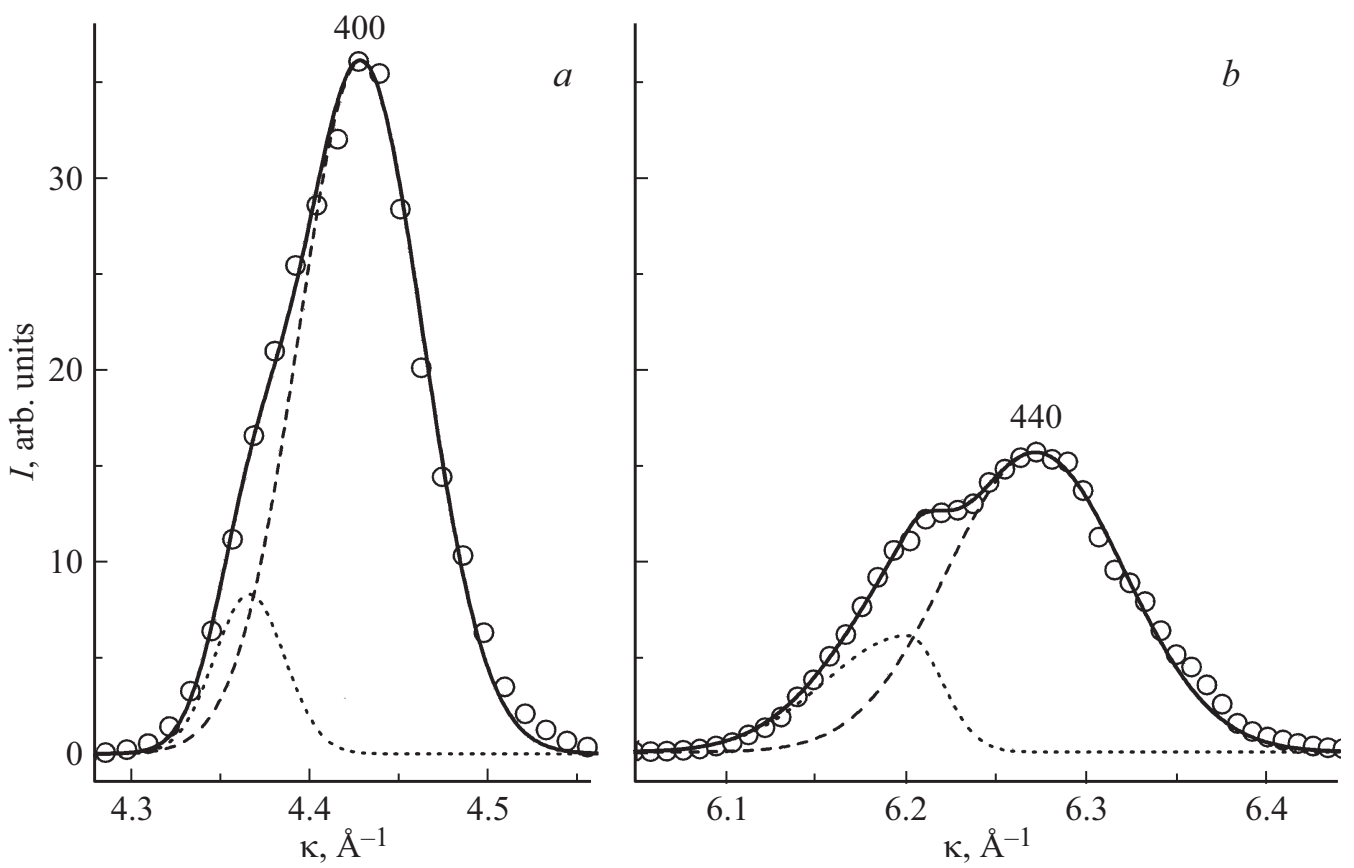

Рис. 4. Картины нейтронной дифракции монокристалла $\mathrm{Zn}_{1-x} \mathrm{Co}_{x} \mathrm{Se}(x=0.15)$, измеренные при $300 \mathrm{~K}$ относительно основных узлов обратной решетки в радиальных направлениях: (400) (a) и (440) $(b)$.

Результаты анализа профилей измеренных в продольных направлениях структурных брэгговских рефлексов кристалла $\mathrm{Zn}_{1-x} \mathrm{Co}_{x} \mathrm{Se}$ $(x=0.15) .\left(\right.$ выборочно $\left.{ }^{*}\right)$

\begin{tabular}{|c|c|c|c|c|c|c|c|c|}
\hline $\begin{array}{c}\text { (Рефлекс) } \\
\text { направление } \\
\text { сканирования }\end{array}$ & $\begin{array}{c}\text { Число } \\
\text { функций } \\
\text { Гаусса } \\
\text { вописании } \\
\text { профиля }\end{array}$ & $\begin{array}{c}\text { Компонент } \\
\text { расщепления }\end{array}$ & $\begin{array}{c}\text { Положение } \\
\text { центра тяжести } \\
\text { рефлекса } \\
\text { (компоненты) } \\
\left|K_{\mathrm{c}}^{\mathrm{t}}\right|, \AA^{-1}\end{array}$ & $\begin{array}{c}\text { Полуширина } \\
\text { рефлекса } \\
\text { FWHM, } \\
\AA^{-1}\end{array}$ & $\begin{array}{c}\text { Вклад } \\
\text { компоненты } \\
\text { в интегральную } \\
\text { интенсивность } \\
I_{\mathrm{I}, \mathrm{i}} / I_{\mathrm{I}, \text { полн }}\end{array}$ & $\begin{array}{c}d_{i}, \\
\AA\end{array}$ & $\begin{array}{c}\Delta d_{i}, \\
\AA\end{array}$ & $\begin{array}{c}\text { Относительная } \\
\text { деформация } \varepsilon \text {, } \\
\%\end{array}$ \\
\hline (111) [111] & 1 & - & 1.920 & 0.043 & - & 3.272 & - & - \\
\hline (200) [100] & 1 & - & 2.210 & 0.047 & - & 2.843 & - & - \\
\hline (220) [110] & 1 & - & 3.130 & 0.068 & - & 2.007 & - & - \\
\hline (222) [111] & 1 & - & 3.830 & 0.093 & - & 1.641 & - & - \\
\hline$(\overline{2} 22)[111]$ & 2 & $\begin{array}{l}\text { больш., симметричн. } \\
\text { меньш., симметричн. }\end{array}$ & $\begin{array}{l}3.828 \\
3.839 \\
3.788\end{array}$ & $\begin{array}{l}0.080 \\
0.065 \\
0.044\end{array}$ & $\begin{array}{l}0.79 \\
0.21\end{array}$ & $\begin{array}{l}1.641 \\
1.637 \\
1.659\end{array}$ & $\begin{array}{c}- \\
0.022\end{array}$ & - \\
\hline (400) [100] & 2 & $\begin{array}{l}\text { больш., симметричн. } \\
\text { меньш., симметричн. }\end{array}$ & $\begin{array}{l}4.421 \\
4.428 \\
4.366\end{array}$ & $\begin{array}{l}0.096 \\
0.082 \\
0.046\end{array}$ & $\begin{array}{l}0.89 \\
0.11\end{array}$ & $\begin{array}{l}1.421 \\
1.419 \\
1.439\end{array}$ & $\begin{array}{c}- \\
0.020\end{array}$ & $\overline{1.4}$ \\
\hline (333) [111] & 2 & $\begin{array}{c}\text { больш., симметричн. } \\
\text { меньш., асимметричн. }\end{array}$ & $\begin{array}{l}5.742 \\
5.760 \\
5.670\end{array}$ & $\begin{array}{l}0.153 \\
0.118 \\
0.074\end{array}$ & $\begin{array}{c}- \\
0.80 \\
0.20\end{array}$ & $\begin{array}{l}1.094 \\
1.091 \\
1.108\end{array}$ & $\begin{array}{c}- \\
0.017\end{array}$ & $\overline{-}$ \\
\hline (440) $[110]$ & 2 & $\begin{array}{c}\text { больш., симметричн. } \\
\text { меньш., асимметричн. }\end{array}$ & $\begin{array}{l}6.252 \\
6.271 \\
6.179\end{array}$ & $\begin{array}{l}0.152 \\
0.116 \\
0.077\end{array}$ & $\begin{array}{c}- \\
0.80 \\
0.20\end{array}$ & $\begin{array}{l}1.005 \\
1.002 \\
1.017\end{array}$ & $\begin{array}{c}- \\
- \\
0.015\end{array} \mid$ & $\begin{array}{l}- \\
- \\
1.5\end{array}$ \\
\hline
\end{tabular}

Пр и ме ч ан и е. * Приведены значения для данных рис. $2-4$. 
кристалла $\mathrm{Zn}_{1-x} \mathrm{Co}_{x} \mathrm{Se}(x=0.15)$ указывает на преобладание деформации растяжения. Величины относительного растяжения оценивались из результатов моделирования профилей дифракционных пиков (узлов обратной решетки, измеренных вдоль радиальных направлений) по разности соответствующих межплоскостных расстояний, определяемых большей и меньшей компонентами расщепления конкретных рефлексов. Как видно из данных таблицы, „дифракционные“ значения относительного растяжения лежат в пределах $1.3-1.5 \%$. Полученная для кристалла $\mathrm{Zn}_{1-x} \mathrm{Co}_{x} \mathrm{Se}(x=0.15)$ нейтронографическая характеристика деформации по порядку величины отвечает аналогичным характеристикам сильно легированных $3 d$-ионами кристаллов халькогенидов цинка, исследованных нами ранее [32-34].

В сопоставлении с результатами наших предыдущих нейтрон-дифракционных исследований [33,35], данные, полученные в настоящей работе, так же демонстрируют признаки сформированных длинноволновых модуляций в РМП с матрицами $\mathrm{ZnSe}$ при повышении уровня легирования $3 d$-примесью. Дополнительные вклады в рассеяние, понимаемые в рамках нетрадиционной кристаллографии как указание на длинноволновую модулированную структуру, обнаружены нами на рефлексе (ㄱ33), соответственно вдоль направления [111], рис. $3, d$. Период модулированной структуры, по проявленным признакам в кристалле $\mathrm{Zn}_{1-x} \mathrm{Co}_{x} \mathrm{Se}(x=0.15)$ при комнатной температуре, составляет $\cong 7.85 \mathrm{~nm}$. При этом моделирование расщепления рефлекса (333) не обнаруживает сателлитов и подразумевает неоднородное поле деформаций. По данным работ $[33,35]$, небольшие различия сателлитов в окрестности структурного рефлекса по интенсивности указывают на слабое отклонение оси длинноволновой модуляции от соответствующего кристаллографического направления (на кристалле $\mathrm{ZnSe}$ c повышенным содержанием хрома эффект был выявлен относительно направления [110], на аналогичных кристаллах с соизмеримым уровнем легирования железом и ванадием — относительно направления [100]). Изменения соотношений интенсивности сателлитов от температуры на дифракционной картине кристалла $\mathrm{Zn}_{1-x} \mathrm{Cr}_{x} \mathrm{Se}$ $(x=0.045)$, показанные в работе [35], являются достаточно ярким свидетельством в пользу того, что проявления длинноволновых модуляций в рассматриваемых кристаллах подвержены сильному влиянию со стороны неоднородного поля деформаций. Следовательно, обнаруженные в настоящей работе различия в моделировании профилей, если они не связаны с симметрийными причинами, подразумевают, что увеличение числа неоднородных деформаций на микроуровне скрывает и/или подавляет признаки длинноволновых модуляций. Сформулированный вывод подкрепляют данные нашего исследования [34], проведенного на серии кристаллов $\mathrm{ZnSe}: \mathrm{V}$, легированных $3 d$-примесью до уровня, близкого к естественному пределу растворимости: признаков длинноволновых модуляций в окрестности структурных рефлексов кристалла с самым высоким содержанием ванадия выявлено не было.

И в заключение отметим, что на картинах нейтронного рассеяния кристалла $\mathrm{Zn}_{1-x} \mathrm{Co}_{x} \mathrm{Se}(x=0.15)$ также детектируются дополнительные отражения ,повернутой“ решетки, соответствующие волновым векторам $k=(1 / 31 / 31 / 3) 2 \pi / a \quad(a-$ параметр кубической элементарной ячейки), которые, согласно детальному рассмотрению в работе [36], могут иметь тройственную природу и являться как следствием двойникования (или формирования еще одной субструктуры), так и признаками состояния, предшествующего концентрационному фазовому переходу.

\section{4. Заключение}

На основе данных проведенного при комнатной температуре нейтронографического исследования охарактеризовано деформированное состояние кубических кристаллов $\mathrm{ZnSe}:$ Со. Установлено, что с повышением содержания примеси возрастает вероятность появления микроблоков, рассеяние на которых подавляет проявление неоднородных нанообластей динамических деформаций, индуцируемых растворенными ионами кобальта. Показано, что на базе областей отдельных атомных смещений при высоком уровне легирования реализуется существенно неоднородная макродеформация растяжения, ее оценки из дифракционных данных составляют значения $1.3-1.5 \%$. При этом, для реальной структуры кристалла $\mathrm{Zn}_{1-x} \mathrm{Co}_{x} \mathrm{Se}(x=0.15)$ экспериментально выявлены признаки того, что на базе составляющих ее повреждений существуют устойчивые возможности к формированию длинноволновых модулированных сверхструктур. Полученные в настоящей работе результаты, по нашему мнению, представляют фундаментальный интерес.

\section{Финансирование работы}

Работа выполнена с использованием Уникальной научной установки (УНУ) „Нейтронный материаловедческий комплекс Института физики металлов“ Уральского отделения РАН („НМК ИФМ“) в рамках государственного задания Минобрнауки России (темы „Поток“, № AАAА-А18-118020190112-8 и „Электрон“ Г.p. № AAAA-A18-118020190098-5).

\section{Конфликт интересов}

Авторы заявляют, что у них нет конфликта интересов.

\section{Список литературы}

[1] Introduction to the Physics of Diluted Magnetic Semiconductors / Eds J. Kossut, J.A. Gaj. Springer Ser. Mater. Sci., 144. Springer (2010). 469 p. 
[2] S.B. Mirov, I.S. Moskalev, S. Vasilyev, V. Smolski, V.V. Fedorov, D. Martyshkin, J. Peppers, M. Mirov, A. Dergachev, V. Gapontsev. IEEE J. Select. Topics Quantum Electron. 24, 1601829: 1-29 (2018).

[3] J.M. Baruah, J. Narayan. Dilute Magnetic Semiconducting Quantum Dots: Smart Materials for Spintronics. Nonmagnetic and Magnetic Quantum Dots / Ed. V.N. Stavrou. IntechOpen (April, 2018). P. 187.

[4] T. Dietl, H. Ohno. Rev. Mod. Phys. 86, 187 (2014).

[5] S. Kumar. Magnetic and Optical Studies of Transition Metal-Doped $\mathrm{ZnS}$ Nanostructures. A diss. Doctor of Philosophy. Faculty of Science Thapar University Patiala, India (2015).

[6] P. Zhang. Magneto-optical Studies of Mn Doped II-VI Group Semiconductor Nanostructures. A diss. Doctor of Philosophy. Faculty of the Graduate School of the University at Buffalo. The State University of N. Y. (2019).

[7] M.T. Pham, N.X. Ca, P.N. Loan, N. Tran, B.T. Huy, N.T. Dang, T.L. Phan. J. Supercond. Nov. Magn. 32, 1761 (2019).

[8] A. Rafiq, M. Imran, M. Aqeel, M. Ikram, H. Majeed, S.G. Hussain, S. Ali. Nanosci. Nanotech. Lett. 11, 1 (2019).

[9] A. Martinez, L. Williams, V. Fedorov, S. Mirov. Opt. Mater. Exp. 5, 558 (2015).

[10] R.W. Stites, S.A. Mc Daniel, J.O. Barnes, D.M. Krein, J.H. Goldsmith, S.Guha, G. Cook. Opt. Mater. Exp. 6, 3339 (2016).

[11] E.J. Turner, S.A. Mc Daniel, N. Tabiryan, G. Cook. Opt. Exp. 27, 12282 (2019).

[12] И.Б. Берсукер. Электронное строение и свойства координационных соединений. Химия, Л. (1976). 352 с.

[13] Магнетохимия. Уч. пособие. Сост. Д.А. Келлерман . Уральский гос. ун-т, Екатеринбург (2008). 156 с.

[14] A. Lewicki, A.I. Schindler, J.K. Furdyna, W. Giriat. Phys. Rev. B 40, 2379 (1989).

[15] T.M. Giebultowicz, P. Klosowski, J.J. Rhyne, T.J. Udovic, J.K. Furdyna, W. Giriat. Phys. Rev. B, 41, 504 (1990).

[16] V.R. Galakhov, T.P. Surkova, M.V. Yablonskikh, A.V. Sokolov, E.Z. Kurmaev, L. Gridneva, S. Bartkowski, M. Neumann, J. Nordgren, S.A. Lopes-Rivera. Phys. Rev. B 68, 033204 (2003).

[17] Полумагнитные полупроводники. / Пер. с англ. под ред. Я. Фурдыны, Я. Косута. Мир, М. (1992). 496 с.

[18] C. Bi, L. Pan, M. Xu, L. Qin, J. Yin. In: Proceedings of $9^{\text {th }}$ IEEE Conf. Nanotechnology (2009). IEEE Nano Organizers 874.

[19] P. Mallikarjuna, J. Sivasankar, M. Rigana Begam, N. Madhusudhana Rao, S. Kaleemulla, J. Subrahmanyam. Mech. Mater. Sci. Eng. J., Magnolithe, 9, 10.2412/mmse90.5.465. hal-01500600 (2017).

[20] L. Hou, L. Pan, B. Liang, Y. Liu, Li Zhang, A. Bukhtiar, L. Shi, R. Liuand, B. Zou. Nanotech. 29, 055707 (2018).

[21] S.T. Pawar, G.T. Chavan, V.M. Prakshale, A. Sikora, S.M. Pawar, S.S. Kamble, N.N. Maldar, L.P. Deshmukh. Mater Sci. Semicond. Proc., 61, 71 (2017).

[22] М.П. Шаскольская. Кристаллография. Высш. шк., М. (1984). $376 \mathrm{c}$

[23] А. Келли, Г. Гровс. Кристаллография и дефекты в кристаллах. Мир, М. (1974). 496 с.

[24] Д.М. Чижиков, В.П. Счастливый. Селен и селениды. Наука, М. (1964). 320 с.

[25] В.С. Урусов, Н.Н. Еремин. Кристаллохимия. Изд-во МГУ, M. (2005). Ч. 2. 125 c.
[26] H. Yuan, L. Kong, T. Li, Q. Zhang. Chin. Chem. Lett. 28, 2180 (2017).

[27] В.И. Максимов, С.Ф. Дубинин, В.Д. Пархоменко, Т.П. Суркова. ФТТ 53, 2093 (2011).

[28] T.P. Surkova, S.F. Dubinin, V.I. Maximov, S.A. Lopez-Rivera. Phys. Status Solidi C 9, 1830 (2012).

[29] М.А. Кривоглаз. Теория рассеяния рентгеновских лучей и тепловых нейтронов реальными кристаллами. Наука, М. (1967) $336 \mathrm{c}$.

[30] Ю.А. Изюмов, В.Е. Найш, Р.П. Озеров. Нейтронография магнетиков. Атомиздат, М. (1981). 194 с.

[31] В.И. Максимов, С.Ф. Дубинин, В.Д. Пархоменко. Поверхность 2, 9 (2013).

[32] T. Surkova, V. Maksimov, S. Dubinin, S.A. Lopez-Rivera. Phys. Status Solidi C 13, 456 (2016).

[33] В.И. Максимов, Т.П. Суркова, В.Д. Пархоменко, Е.Н. Юшкова. ФТТ 58, 4, 633 (2016).

[34] В.И. Максимов, Е.Н. Максимова, Т.П. Суркова. ФТТ 60, 1, 50 (2018).

[35] В.И. Максимов, С.Ф. Дубинин, Т.П. Суркова, А.В. Королёв. ФТТ 55, 10, 1912 (2013).

[36] В.И. Максимов, Е.Н. Максимова, Т.П. Суркова, А.П. Вохмянин. ФТТ 61, 1, 42 (2019).

Редактор Т.Н. Василевская 